\title{
Sensitivity of the Inhomogeneous Magnetization Transfer Imaging Technique to Spinal Cord Damage in Multiple Sclerosis
}

(D) H. Rasoanandrianina, (DS. Demortière, (D) A. Trabelsi, (D).P. Ranjeva, (D). Girard, (D) G. Duhamel, (D). Guye, (D). Pelletier,

(D) B. Audoin, and (D) V. Callot

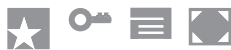

\begin{abstract}
BACKGROUND AND PURPOSE: The inhomogeneous magnetization transfer technique has demonstrated high specificity for myelin, and has shown sensitivity to multiple sclerosis-related impairment in brain tissue. Our aim was to investigate its sensitivity to spinal cord impairment in MS relative to more established MR imaging techniques (volumetry, magnetization transfer, DTI).
\end{abstract}

MATERIALS AND METHODS: Anatomic images covering the cervical spinal cord from the $\mathrm{Cl}$ to $\mathrm{C} 6$ levels and DTI, magnetization transfer/inhomogeneous magnetization transfer images at the C2/C5 levels were acquired in 19 patients with MS and 19 paired healthy controls. Anatomic images were segmented in spinal cord $G M$ and WM, both manually and using the $A M U_{40}$ atlases. MS lesions were manually delineated. MR metrics were analyzed within normal-appearing and lesion regions in anterolateral and posterolateral WM and compared using Wilcoxon rank tests and $z$ scores. Correlations between MR metrics and clinical scores in patients with MS were evaluated using the Spearman rank correlation.

RESULTS: $\mathrm{AMU}_{40}$-based Cl-to-C6 GM/WM automatic segmentations in patients with MS were evaluated relative to manual delineation. Mean Dice coefficients were $0.75 / 0.89$, respectively. All MR metrics (WM/GM cross-sectional areas, normal-appearing and lesion diffusivities, and magnetization transfer/inhomogeneous magnetization transfer ratios) were observed altered in patients compared with controls $(P<$ .05). Additionally, the absolute inhomogeneous magnetization transfer ratio $z$ scores were significantly higher than those of the other MR metrics $(P<.0001)$, suggesting a higher inhomogeneous magnetization transfer sensitivity toward spinal cord impairment in MS. Significant correlations with the Expanded Disability Status Scale $(\rho=-0.73 / P=.02, \rho=-0.81 / P=.004)$ and the total Medical Research Council scale $(\rho=0.80 / P=.009, \rho=-0.74 / P=.02)$ were observed for inhomogeneous magnetization transfer and magnetization transfer ratio $z$ scores, respectively, in normal-appearing WM regions, while weaker and nonsignificant correlations were obtained for DTI metrics.

CONCLUSIONS: With inhomogeneous magnetization transfer being highly sensitive to spinal cord damage in MS compared with conventional magnetization transfer and DTI, it could generate great clinical interest for longitudinal follow-up and potential remyelinating clinical trials. In line with other advanced myelin techniques with which it could be compared, it opens perspectives for multicentric investigations.

ABBREVIATIONS: $\mathrm{AMU}=$ Aix-Marseille University; CSA = cross-sectional areas; EDSS = Expanded Disability Status Scale; FA $=$ fractional anisotropy; HC $=$ healthy controls; ihMT = inhomogeneous magnetization transfer; ihMTR = inhomogeneous magnetization transfer ratio; $\lambda_{/ /}=$axial diffusivity; $\lambda_{\perp}=$ radial diffusivity; MRC = Medical Research Council; MT = magnetization transfer; MTR = magnetization transfer ratio; NA = normal-appearing; SC = spinal cord; TWT $=$ Timed 25-Foot Walk Test; SCT $=$ Spinal Cord Toolbox

$\mathrm{S}$ is a chronic autoimmune disease of the CNS affecting the brain and the spinal cord (SC), characterized by multifocal

Received June 20, 2019; accepted after revision March 12, 2020

From the Center for Magnetic Resonance in Biology and Medicine (H.R., S.D., A.T., J.P.R., O.G., G.D., M.G., J.P., B.A., V.C.), Centre National de la Recherche Scientifique, Aix-Marseille Université, Marseille, France; Centre d'Exploration Métabolique par Résonance Magnétique (H.R., S.D., A.T., J.P.R., O.G., G.D., M.G., J.P., B.A., V.C.), Assistance Publique-Hopitaux de Marseille, Hôpital Universitaire Timone, Marseille, France; Laboratoire de Biomécanique Appliquée, Institut Français des Sciences et Technologies des Transports, de l'Aménagement et des Reseaux, Aix-Marseille Université; iLab-Spine International Associated Laboratory (H.R., J.P.R., V.C.), Marseille-Montreal, France-Canada; and Department of Neurology (S.D., J.P., B.A.), Centre Hospitalier Universitaire Timone, Assistance Publique-Hopitaux de Marseille, Marseille, France.

Henitsoa Rasoanandrianina and Sarah Demortière contributed equally to this article. demyelination and inflammation, edema, axonal loss, and gliosis, whose diagnosis is based on anatomic MR imaging. ${ }^{1}$ Although

This work was performed by a laboratory member of France Life Imaging network (grant ANR-11-INBS-0006) and supported by the French Centre National de la Recherche Scientifique, the French Ministère de l'Enseignement Supérieur et de la Recherche, and the Fondation pour l'Aide à la Recherche sur la Sclérose En Plaques.

Please address correspondence to Virginie Callot, PhD, CRMBM-CEMEREM, UMR 7339, CNRS, Aix-Marseille Université, Faculté de Médecine, 27, bd Jean Moulin, 13385 Marseille Cedex 5, France; e-mail: virginie.callot@univ-amu.fr

- Indicates open access to non-subscribers at www.ajnr.org

E Indicates article with supplemental on-line tables.

Indicates article with supplemental on-line photo

http://dx.doi.org/10.3174/ajnr.A6554 
extensive investigations including demonstration of multicentric capabilities and scan time reduction are still necessary to bridge the gap to clinical practice, quantitative MR imaging techniques now have the potential to help improve disease diagnosis and monitoring, ${ }^{2}$ as well as treatment evaluation.

Given the clinical relevance and the risk of long-term disability in spinal $\mathrm{MS}^{3}{ }^{3}$ a better characterization of pathologic mechanisms underlying SC tissue impairment, including myelin damage, is therefore essential.

Unfortunately, SC MR imaging lags behind brain MR imaging, mainly due to technical challenges related to the small size, CSF pulsatility, respiratory motion, and susceptibility artifacts of the spinal cord due to surrounding bony structures. ${ }^{4}$ Nonetheless, due to recent methodologic improvements, a growing number of SC MR imaging studies in MS have been reported, mostly using $\mathrm{DTI}^{5}$ and conventional magnetization transfer $(\mathrm{MT})^{6}$ imaging, highlighting SC microstructural changes with significant correlations to physical disability. $^{7,8}$

Although highly sensitive to MS-related tissue changes, both DTI and MT metrics are not specific to myelin damage because they could also be affected by inflammation and/or axonal loss. ${ }^{9}$ Tremendous effort has thus recently been made toward the development of more myelin-specific MR imaging techniques. ${ }^{10,11}$ These particularly include myelin-water imaging, ${ }^{12}$ which gives access to the myelin water fraction ${ }^{13}$ or water protons associated with myelin trapped between the finely spaced lipid bilayers of the myelin sheaths, as well as quantitative MT, ${ }^{14,15}$ which gives a robust estimate of the macromolecular-to-free pool size ratio, which has been shown to strongly correlate with white matter myelin density, ${ }^{16}$ or the recently described inhomogeneous MT (ihMT) technique. ${ }^{17-19}$ This latter allows selectively isolating the contribution of dipolar order within the broad MT signal and hence appears particularly sensitive to myelinated structures due to their relatively long dipolar relaxation times. ${ }^{17}$ Applied in a preliminary clinical MS study, ihMT was found to be highly sensitive to cerebral microstructural changes with strong correlation to the Expanded Disability Status Scale (EDSS).$^{20}$ Furthermore, embedded within a protocol optimized for the $\mathrm{SC},{ }^{18}$ it has also already demonstrated high sensitivity to SC tissue changes in both aging healthy subjects ${ }^{21}$ and patients with amyotrophic lateral sclerosis. $^{22}$

In line with these studies, the main purpose of this work was to assess the sensitivity of the ihMT technique to SC impairment in MS relative to more conventional MR imaging markers, including atrophy, MT, and DTI measures and to demonstrate its potential in clinical monitoring. To do so, we applied a multiparametric MR imaging protocol, including high-resolution anatomic and quantitative DTI and MT/ihMT imaging techniques, together with a SC template-based approach to quantify data collected in patients with MS and age-matched healthy controls (HC) in different ROIs.

\section{MATERIALS AND METHODS \\ Subjects}

This retrospective study was approved by our institutional ethics committee (CCP Sud Méditerranée 1), and written informed
Table 1: Demographic and clinical features of the study population including $\mathrm{HC}$ and patients with $\mathrm{MS}^{\mathrm{a}}$

\begin{tabular}{lcc}
\hline \multicolumn{1}{c}{ Demographics } & HC & MS \\
\hline Count (female/male) & $19(11 / 8)$ & $19(11 / 8)$ \\
Age (years) & $52(22-74)$ & $46(26-70)$ \\
Disease duration (months) & - & $117(48-372)$ \\
EDSS & - & $2.5(1-7)$ \\
Total MRC ${ }^{\mathrm{b}}$ (/120) & - & $118(103-120)$ \\
NHPT $^{\mathrm{c}}(\mathrm{sec})$ & - & $24.1(17.8-73.4)$ \\
TWT $(\mathrm{sec})^{\mathrm{N}}$ & - & $5.5(3.4-73)$ \\
Handgrip $^{\mathrm{d}}(\mathrm{kPa})$ & - & $82.5(25.5-122.5)$ \\
No. of SC lesions & - & $4(2-7)$ \\
Volume of brain lesions $\left(\mathrm{mm}^{3}\right)$ & - & $3261(726-38,013)$ \\
\hline
\end{tabular}

Note:-NHPT indicates Nine-Hole Peg Test; -, not applicable measurements.

${ }^{a}$ Reported values are medians (minimum-maximum). Lesion burden in the cervical SC and brain of patients with MS is also reported.

${ }^{b}$ Medical Research Council scale: normal value per muscle, 5; range, 0-5, for upper (7 muscles on each side) and lower limbs (5 muscles on each side), with a total maximum score of 120 .

${ }^{\mathrm{c}}$ The Nine-Hole Peg Test for each hand and TWTs were performed twice for each patient, and the best performance was preserved.

${ }^{d}$ Handgrip measurements for each patient were repeated thrice for each hand and then averaged to get 1 value per limb.

consent was obtained from all subjects. Nineteen patients with MS (13 relapsing-remitting and 6 secondary-progressive forms) and 19 sex- and age-matched HC were included during 2 years. Inclusion criteria for patients were a diagnosis of MS according to the revised McDonald criteria ${ }^{23}$ and clinical symptoms suggesting SC involvement. Clinical symptoms were evaluated $>3$ months after steroid infusion. Exclusion criteria were any neurologic disease other than MS and chronic psychiatric diseases. Neurologic examinations were performed right after the MR imaging acquisitions. They included a specific MS global disability rating scale with EDSS scoring. A muscle strength grading with the Medical Research Council scale (MRC) ${ }^{24}$ for the 4 limbs was also performed. Specific assessment of the upper limbs was performed with a handgrip strength test ${ }^{25}$ and the Nine-Hole Peg Test, currently used in MS to assess manual dexterity. For lower limb evaluation, a Timed 25-Foot Walk Test (TWT) ${ }^{26}$ was performed. Additional details are found in Table 1.

\section{Image Acquisition}

MR imaging was performed on a 3T scanner (Magnetom Verio; Siemens) using the body coil for transmission and standard 12channel head, 4-channel neck, and 24-channel spine matrix array coils for reception. The subjects were positioned with the neck in a neutral position using a cervical collar to reduce subject motion.

The MR imaging protocol (summarized in On-line Table 1) was optimized to last approximately 50 minutes. It included a 3D-T2WI sequence for vertebral level identification and cervical lesion depiction (see postprocessing in Fig 1), a 2D multisection multiangle T2*WI multiecho gradient-echo sequence ( 7 slices, 1 per cervical level from $\mathrm{C} 1$ to $\mathrm{C} 7)$, providing a good contrast to delineate GM and WM, as well as 2-level DTI and ihMT acquisitions (single-section) centered at mid-C2 and mid-C5 cervical levels, placed strictly perpendicular to the cord axis. Patients also underwent, during the same session, conventional brain and SC acquisitions at the cervical level (T1WI, T2WI, FLAIR, STIR, and postgadolinium T1) for lesion burden evaluation. 


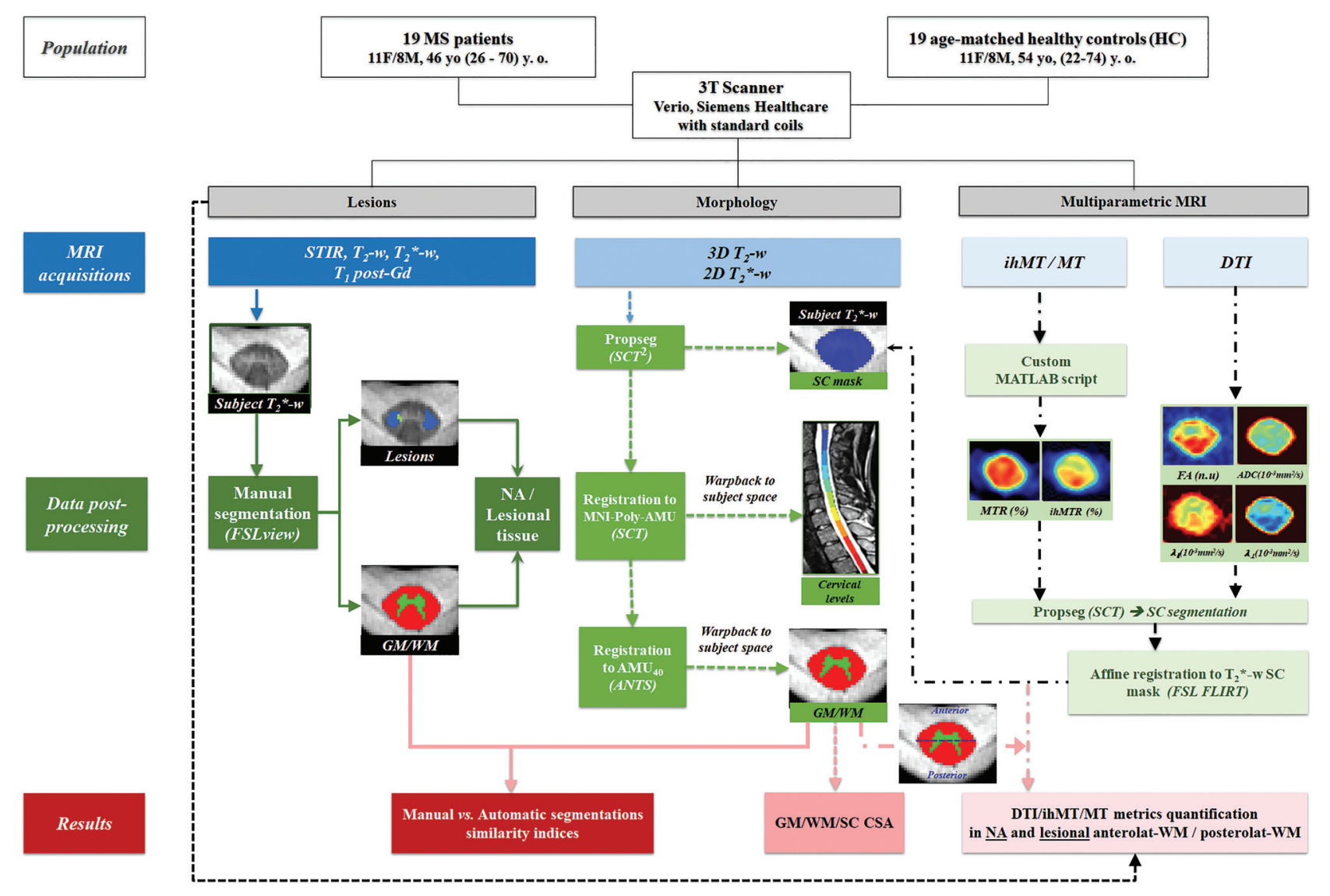

FIG 1. Design of the study. Acquisition and postprocessing pipeline for MR imaging data shown for the C2 cervical level in a patient with MS, including the following: manual SC, GM/WM, and lesional/NA tissue segmentations on T2*WI and registration of the 3D-T2WI to the MNI-PolyAMU template to automatically identify the cervical levels from $\mathrm{Cl}$ to $\mathrm{C} 7$ (warped back into the subject's space; this step allows cord volume per level measurements, not reported in this study); SC segmentation on T2*WI and T2WI by the SCT PropSeg function; T2WI registration to the MNI-Poly-AMU template ${ }^{28}$ to obtain cervical levels; $\mathrm{T2}^{*} \mathrm{WI}$ registration to the $\mathrm{AMU}_{40}$ template ${ }^{29}$ to obtain $\mathrm{GM} / \mathrm{WM}$ segmentations, warped back into the subject's space followed by CSA (square millimeters) extraction and intrasubject multiparametric data normalization, taking into account the presence of lesions, allowing metrics extraction from lesional and NA anterolateral WM and posterolateral WM, derived from the WM tracts atlas and defined as delimited by the blue horizontal line. Masks were eroded (2D surface) before quantification. y.o. indicates years of age; Gd, gadolinium; anterolat, anterolateral; posterolat, posterolateral; FSL view refers to the FSL viewer software; ${ }^{30}$ ANTs, Advanced Normalization Tools (http:// stnava.github.io/ANTs/); FLIRT, FMRIB is the FSL Linear Image Registration Tool (http://www.fmrib.ox.ac.uk/fsl/fslwiki/FLIRT); ${ }^{51}$ WM tracts atlas (https://sourceforge.net/p/spinalcordtoolbox/wiki/White\%20Matter\%20atlas/). ${ }^{32}$

\section{Data Analysis}

The entire postprocessing pipeline, detailed in Fig 1, was performed off-line using a custom Matlab script (Matlab R2014a; MathWorks), relying on the Spinal Cord Toolbox (SCT; https:// sourceforge.net/projects/spinalcordtoolbox/) ${ }^{27}$ functions and dedicated SC templates and atlases (MNI-Poly-AMU; https:// sourceforge.net/p/spinalcordtoolbox/wiki/MNI-Poly-AMU/) ${ }^{28}$ and $\mathrm{AMU}_{40}{ }^{29}$ ), allowing automatic delineation of the GM and WM.

To evaluate the efficacy of the $\mathrm{AMU}_{40}$-based automated GM/ WM segmentation applied for the first time in MS, 2 independent experts (S.D., an in-training neurologist, and H.R., a 3-year MR imaging expert) manually outlined the SC and GM using FSLview software (Version 5.0.10; http://fsl.fmrib.ox.ac.uk/fsl/ fslwiki/fslview $/)^{30}$ on all T2*WI images. The WM region was subsequently obtained by subtracting SC and GM masks. Agreement between the 2 raters and between manual and automatic segmentations was evaluated by computing 3 conventional parameters: ${ }^{31}$ the Dice similarity coefficient (range, 0-1, with 1 indicating perfect similarity), the Haus dorff distance (in millimeters), and the maximum median distance between skeletonized segmentations (in millimeters), using the SCT. ${ }^{27}$ For each subject, a comparison of the $3 \mathrm{WM} / \mathrm{GM}$ metrics between the 2 raters was performed; then, the average value per structure was calculated. All automatic segmentations presenting manual/automatic Dice coefficients of $<0.8$ (considered imperfect) were manually corrected under strict consensus between the 2 experts and subsequently used for multiparametric metrics quantification.

Morphologic measurements were then performed on all subjects by extracting the cross-sectional areas (CSA, in square millimeters) from automatic GM/WM segmentations (manually corrected if necessary) at each cervical level from C1 to C6 (C7 was discarded due to subject motion in 7/19 subjects [37\%]).

Cervical SC lesions (characterized by signal hyperintensities) were finally depicted and counted from both STIR and T2WI. Then, for quantitative analyses, manual segmentation of lesions was performed on the T2*WI by expert 1 (S.D.) using both STIR and T2WI series as additional references to improve the accuracy 
of the lesion location. All the segmented lesions were visible on all 3 images. Normal-appearing (NA) regions were subsequently differentiated from lesion regions for metrics quantification.

For quantitative analysis, 4 DTI metrics (fractional anisotropy [FA], ADC, axial diffusivity $\left[\lambda_{/ /}\right]$, and radial diffusivity $\left[\lambda_{\perp}\right]$, along with MT and ihMT ratios [MTR/ihMTR]) were derived afterward and quantified within the anterolateral WM and posterolateral WM at C2 and C5. The GM was not considered. These regions were chosen to comply with current ihMT HASTE limitations in terms of partial volume effect and the loss of spatial resolution (see Discussion). To match ihMT single-section quantification, only the middle section was considered for the DTI metrics. At the end of the postprocessing pipeline, the quantitative dataset for each subject included SC, GM, and WM CSA at each level from $\mathrm{C} 1$ to $\mathrm{C} 6$, as well as mean \pm SD values of FA, ADC, $\lambda_{\perp}, \lambda_{/ /}$, ihMTR, and MTR from anterolateral and posterolateral WM regions free from or having MS lesions (NA/lesional regions). For each metric, values from C2 and C5 were averaged to obtain a single value per ROI per subject for statistical purposes.

Last, brain lesions were manually segmented on T2 and FLAIR images to estimate their volume.

\section{Statistical Analysis}

Statistical analyses were performed using JMP9 (SAS Institute), considering $P<.05$ for statistical significance.

\section{Comparison of MR Imaging Data between Patients with MS and Healthy Controls}

For MS-related morphologic change evaluation, GM, WM, and SC CSA at each level were compared between patients with MS and $\mathrm{HC}$, using nonparametric Wilcoxon rank tests corrected for multiple comparisons ( $n=6$ cervical levels) with the HolmBonferroni method.

Considering multiparametric data, we used the nonparametric Steel-Dwass all-pairs tests corrected for multiple comparisons to assess DTI/MT/ihMT metrics differences between patients and $\mathrm{HC}$.

\section{Sensitivity of ihMT to MS-Related Changes}

DTI, MT, and ihMT metrics $z$ scores were computed for NA and lesional anterolateral WM, posterolateral WM, and total WM for each patient with MS relative to mean values of HC. CSA $z$ scores, all cervical levels considered, were calculated for global GM, WM, and SC.

$Z$ scores of ihMTR were then compared with $z$ scores of all metrics using nonparametric Wilcoxon rank tests to assess the sensitivity of ihMT to MS-related changes compared with the more conventional atrophy (CSA), DTI, and MT measures. Henceforth, the term "metric variation" will be used to refer to a change in the absolute $z$ score value of the metrics.

Correlations with Clinical Scores. Correlations among brain lesion volume, SC lesion number, SC/GM/WM CSA, and DTI/ MT/ihMT metrics variations (ie, $z$ scores) in lesion and NA WM regions and each clinical score (disease duration, total MRC, handgrip, TWT, Nine-Hole Peg Test, and EDSS) were evaluated using the statistical nonparametric Spearman rank test. The resulting significant correlations were then corrected to control for multiple comparisons using the false discovery rate procedure.

\section{RESULTS}

\section{Patient Characteristics}

Demographic information and clinical features of patients with MS are summarized in Table 1. The median EDSS score and disease duration of the patients at the time of MR imaging were 2.5 and 117 months, respectively.

\section{Reliability of the Automated $\mathrm{AMU}_{40} \mathrm{GM} / W M$ Segmentation}

On-line Table 2 summarizes the similarity indices (Dice coefficient, Hausdorff, and skeletonized mean distances) with regard to manual GM/WM segmentations. The reader is referred to the On-line Figure for illustrations of representative $\mathrm{AMU}_{40}$-based $\mathrm{GM}$ segmentations from patients with MS with/without the presence of lesions. In the $\mathrm{HC}$ group, $\mathrm{AMU}_{40}$-based segmentations were found to be highly reliable with mean Dice coefficients of $0.85 \pm 0.05$ and $0.92 \pm 0.02$ for GM and WM, respectively. In patients with MS, 8/114 slices were discarded due to visually observed poor contrast in the images, mostly due to breathing motion of the patients. When applied to the remaining 106 slices, indices from WM (Dice, $0.89 \pm 0.04$ ) were close to those found in HC, whereas the values from GM segmentations $(0.75 \pm 0.14)$ were found to be lower, with higher SDs. Worse GM indices were found in slices with lesions $(0.73 \pm 0.12)$ compared with slices without lesions $(0.79 \pm 0.09)$, whereas WM indices were similar whether or not lesions were present in the slices. Afterward, 29\% (31/106 slices) of the $\mathrm{AMU}_{40}$-based segmentations (with Dice, $<0.8$ ) were manually corrected for measurements of CSA and multiparametric analysis.

\section{Comparison of MR Imaging Data between Patients with MS and Healthy Controls}

Boxplots of GM and WM CSA from HC and patients with MS along with the corresponding $z$ scores and percentages of variation are presented in Fig 2. A significant decrease in CSA for the SC $(P<.001$, data not shown $)$ and WM $(P<.003)$ across all cervical levels was found in patients with MS compared with HC. Decrease trends were additionally found for GM areas, significant at C6 $(P=.003)$.

Figure 3 shows representative multiparametric datasets of 2 patients with MS (MS_1: 64 years of age, EDSS score, 2; MS_2: 60 years of age, EDSS score, 6) and a healthy control (65 years of age). Overall, in patients with MS, the DTI, ihMTR, and MTR values were all found altered in both NA and lesional WM regions (Table 2 and Fig 4) compared with the healthy control. $\lambda_{\perp}$ and ADC showed trends toward an increase, albeit not significant, whereas ihMTR and MTR significantly decreased in both regions, with higher metric variations found in regions with lesions compared with NA tissue.

\section{Sensitivity of ihMT to MS-Related Changes}

Among all DTI, MT, and ihMT metrics, ihMTR showed the most important metrics variations (ie, higher absolute $z$ score values) as illustrated in Fig 4. Results of Wilcoxon rank tests showed significant differences between these ihMTR variations and $z$ scores 
Healthy controls $(n=19)$
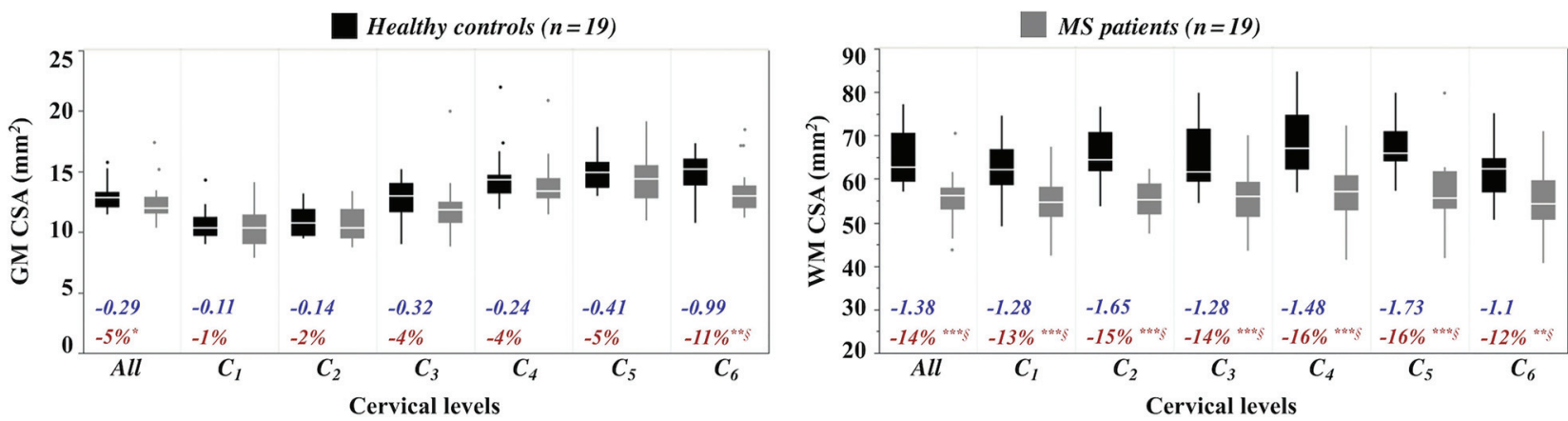

FIG 2. CSAs (square millimeters) in $\mathrm{HC}$ and patients with MS. Mean values were extracted from $\mathrm{AMU}_{40}$-based $\mathrm{GM}$ and $\mathrm{WM}$ segmentations for each cervical level ( $\mathrm{Cl}$ to $\mathrm{C} 6)$. Boxplots of subjects' mean CSA values across all cervical levels are also presented in the first column of each graph. The boxplots represent the minimum, first quartile (25\%), the median, the third quartile (75\%) and the maximum from upper to lower, respectively. Significant decreases in CSA in patients with MS relative to HC, assessed using Wilcoxon rank tests are reported as ${ }^{\star} P<.05,{ }^{* \star} P<.001,{ }^{\star \star *} P<.0001$. The section symbol indicates statistical differences that survive the Holm-Bonferroni correction for multiple comparisons. The percentage difference of CSA between MS and HC groups (in red) and the average $z$ scores (no unit, in blue) in patients with MS relative to $\mathrm{HC}$ at each level are also reported; a negative value indicates a decrease in CSA in patients.
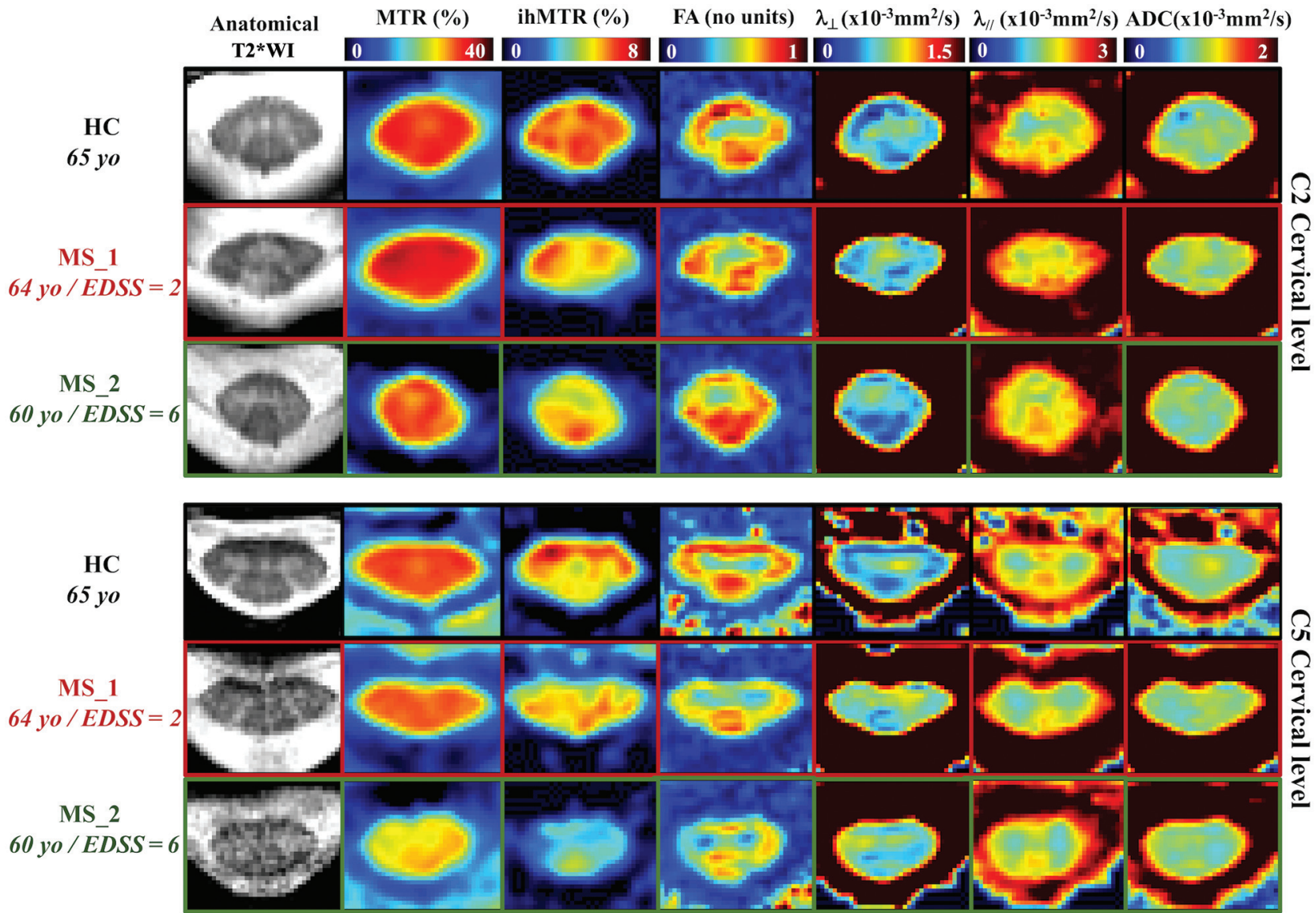

FIG 3. Representative multiparametric maps extracted from a patient with relapsing-remitting MS (MS_1, 64 years of age and EDSS score, 2) and a patient with secondary-progressive MS (MS_2, 60 years of age and EDSS score, 6) along with maps from an age-matched HC (65years of age) for illustration. yo, years of age.

of DTI and MT metrics $(P<.0001)$, in both NA and lesional regions

\section{Correlations with Clinical Scores}

Significant correlations were found between ihMTR $z$ scores in NA whole-WM and total MRC scores $(\rho=0.80, P=.009)$ as well as EDSS scores $(\rho=-0.73, P=.02)$. MTR $z$ scores were also found correlated with total MRC scores $(\rho=0.74$, $P=.02)$ and EDSS scores $(\rho=-0.81, P=.004)$. On the other hand, weaker and nonsignificant correlation trends were found for DTI metrics variations, such as between FA $z$ scores and MRC scores $(\rho=0.64, P=.06)$, for example. 
Brain lesion volume was also significantly correlated with the TWT $(\rho=0.66, P=.008)$. Because correlations with the TWT were mostly related to data from a patient with important clinical disabilities (TWT $=73$ seconds), they were not considered afterward. No other significant correlations were found.

\section{DISCUSSION}

This study provides 3 main findings: 1) $\mathrm{AMU}_{40}$-based SC WM/ GM segmentation is feasible in patients with MS; 2) ihMTR shows higher sensitivity to MS-related SC pathophysiologic changes compared with more conventional approaches; and 3) ihMTR might be useful in a clinical context.

MR templates and atlases are very useful tools for automated postprocessing pipelines. Across the years, effort has been directed toward construction ${ }^{28,29,32,33}$ and application of such templates in the SC. ${ }^{29,34}$ To our knowledge, only 2 studies $^{35,36}$ applied and evaluated automated SC GM segmentation in MS: the work of Prados et $\mathrm{al}^{35}$ based on WM/GM segmentation using the Similarity and Truth Estimate for Propagated Segmentations algorithm and the work of Datta et al, ${ }^{36}$ based on active contour segmentation, with both studies mostly focusing on the C2/C3 cervical levels. Our work is the first to apply the $\mathrm{AMU}_{40}$ template in SC MS and investigate WM/GM segmentation capability from $\mathrm{C} 1$ to $\mathrm{C} 6$ cervical levels in such cases. The $\mathrm{AMU}_{40}$-based segmentation was previously validated in healthy subjects ${ }^{21}$ (with Dice coefficients of $\sim 0.90 / 0.83$ for $\mathrm{WM} / \mathrm{GM}$, respectively) and was used in patients with amyotrophic lateral sclerosis. ${ }^{22}$ In this study, WM/ GM segmentation in MS seemed less efficient than in HC, mostly due to the presence of lesions extending on

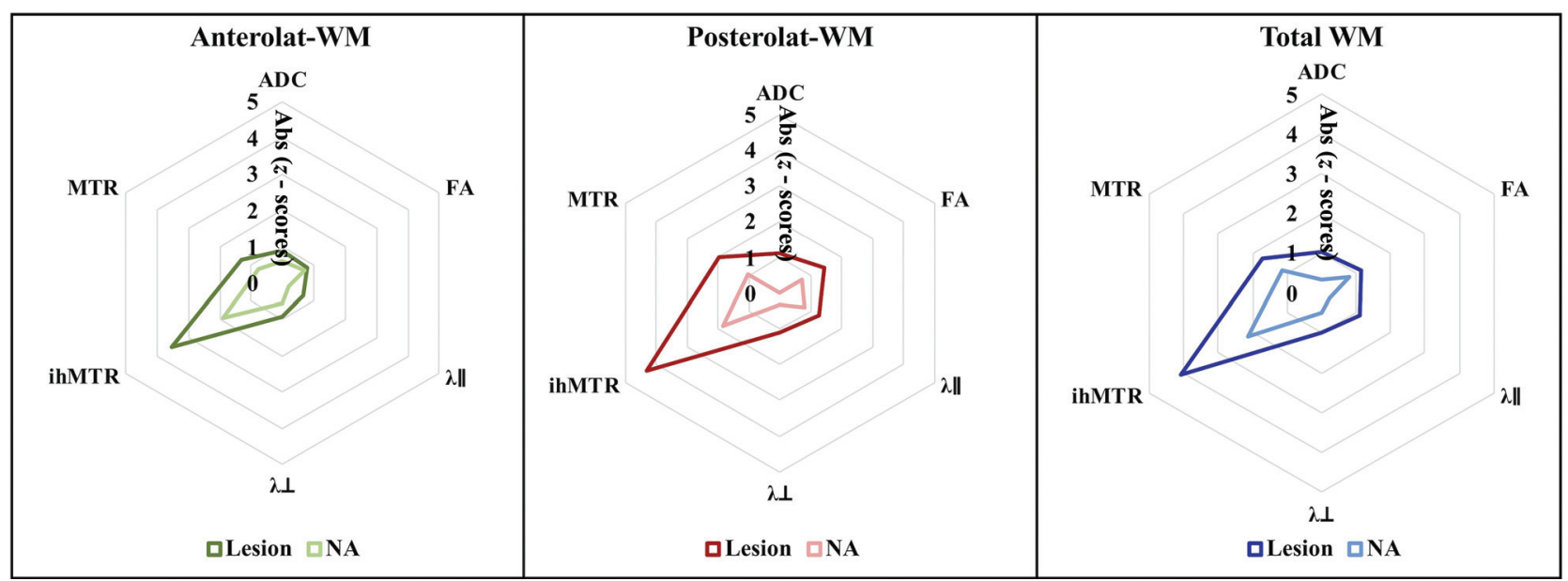

FIG 4. Spider graphs showing mean absolute $z$ scores of each quantitative MR metric in patients with MS relative to HC computed for NA and lesional anterolateral WM (left graph), posterolateral WM (middle graph), and total WM (right graph). Wilcoxon tests for paired values performed between ihMTR absolute $z$ scores and each of the other metrics to assess the sensitivity of the ihMT technique to microstructural changes in MS compared with DTI and MT show significant differences of all tested pairs, with $P<.0001$. Absolute (Abs) $z$ scores are used for visualization purposes and statistical comparison. 
both GM and WM, mitigating the difference of contrasts between these structures. The template, nonetheless, demonstrated fairly good compliance with manual segmentations, with Dice coefficients $(0.76 \pm 0.09$ at $\mathrm{C} 2 / \mathrm{C} 3)$ in line with the 2 previously mentioned MS studies, including patients with visible lesions (GM Dice coefficients: $0.80 \pm 0.07$ and $0.88 \pm 0.03$, respectively, at $\left.\mathrm{C}_{2-3}\right) .{ }^{35,36}$ The $\mathrm{AMU}_{40}$ template is not available for whole-spinal cord coverage or for $3 \mathrm{D}$ data; however, a former version of it $\left(\mathrm{AMU}_{15}\right)$ could be found in the recently proposed PAM50 ${ }^{33}$ template (formerly the MNI-Poly-AMU template; https://sourceforge.net/p/spinalcordtoolbox/wiki/MNIPoly-AMU/), which presents the advantages of being compatible with larger spatial coverage, 3D acquisitions, and acquisition with different contrasts, while also proposing segmentation approaches based on machine learning. Comparison with this template version was beyond the scope of the study but would be interesting.

MS-related morphologic changes have been largely reported with evidence of cervical SC and GM atrophy in patients with MS relative to $\mathrm{HC}$, with a strong correlation with clinical disability. ${ }^{37-}$

${ }^{39}$ In our study, SC, WM, and GM semiautomated measurements of CSA were performed along the cervical cord from $\mathrm{C} 1$ to $\mathrm{C} 6$ and results suggested atrophy of both SC GM and WM in patients with MS (5\%/14\% decrease on average, respectively), consistent with the literature, with more important and more significant atrophy in WM.

DTI and MT imaging have already been largely used to evaluate MS-related SC microstructural changes, with reports of higher ADC and $\lambda_{\perp}$ and lower FA and MTR in patients with MS relative to HC. ${ }^{7,40,41}$ In this study, DTI and MT metrics in patients with MS demonstrated overall similar variations, with higher variations in lesions compared with NA tissue, suggesting MSrelated microstructural changes in the cervical SC as described in previous reports. However, both DTI and conventional MT techniques have been proved to lack specificity toward any pathophysiologic mechanism, due to confounding factors linked to inflammation, demyelination, edema, or axonal degeneration, ${ }^{9}$ making the use of more myelin-specific or sensitive methods interesting.

This work focused on ihMT. Previously published studies reported a supposedly higher ihMT specificity to myelinated structures compared with conventional MT as well as higher sensitivity o associated microstructural changes, in both healthy ${ }^{21}$ and pathologic SC (amyotrophic lateral sclerosis ${ }^{22}$ ), and in the pathologic (MS) brain. ${ }^{20}$ In the present SC study, among all the considered MR metrics (CSA, DTI, ihMTR, and MTR), ihMTR had the most important and significant variations (absolute value decreases compared with HC and highest $z$ scores), particularly in lesions. Although less important than in lesions, an ihMTR decrease in NA tissue of patients with MS was also found highly significant, suggesting tissue changes occurring away from a lesion or before lesion occurrence, currently not visible on conventional MR imaging. Better detection of such subtle changes could improve disease monitoring, particularly for future treatment based on neuroprotection. Of interest and though not assessed in the current MS work, the ihMT technique was previously demonstrated to be highly reproducible, with a small scanrescan ihMTR variability $(2.9 \%) .^{21}$
Finally, despite a readout module inducing severe blurring and precluding quantification within specific WM tracts or GM areas, ihMTR $z$ scores measured in total NA WM regions were significantly associated with MRC and EDSS scores, whereas correlations between MTR and DTI with most clinical scores were not significant. These results could advocate for clinical and trial applications of the ihMT technique. They also support the higher sensitivity of the ihMT to MS-related deficits compared with DTI or MT. Further investigations related to the ihMT myelin specificity should now be performed, in conjunction with other myelin imaging techniques that have been successfully applied to SC imaging in recent years, such as quantitative MT imaging ${ }^{15}$ or myelin water fraction, which have already demonstrated great interest for SC MS investigations. ${ }^{15,42}$ Future studies should also focus on the ability to detect subtle and early changes.

Indeed, from the clinical point of view, in addition to the small number of patients, the main limitations were the cohort heterogeneity (especially regarding phenotypes, combining relapsing-remitting and secondary-progressive) and the disease duration. It would be relevant to include early forms of MS for longitudinal monitoring of ihMTR variation at different times to judge its utility to better characterize myelin-related processes. From the methodologic point of view, the readout module used for the ihMT measurements induced severe resolution loss and tissue blurring ( $\sim 6$ voxels for a T2 of $75 \mathrm{~ms}$ ) in the phase-encoding direction (right-left), hence only allowing investigating anterolateral and posterolateral regions free from lesions or presenting lesions. Further acquisition strategies, such as recently presented, ${ }^{43,44}$ should help in achieving refined tissue separation. These latter strategies should also permit investigating multiple SC levels and hence characterize the diffuse pattern of cervical SC tissue alteration in MS (DTI/MT/ ihMT acquisitions were limited here to C2 and C5 levels to keep the protocol duration to $<1$ hour). Optimized excitation pulse schemes and translation to higher fields (eg, $7 \mathrm{~T}^{45,46}$ ) should also be considered to increase spatial resolution, SNR, and sensitivity. ${ }^{47}$ Finally, lesions were manually delineated; further studies should evaluate the use of the recently proposed automated MS lesion segmentation algorithms using convolutional neural networks ${ }^{48,49}$ or lesion-prediction algorithms.

Nevertheless, despite these limitations, the MR imaging acquisition and template-based postprocessing strategies used in the current study led to promising results supporting higher ihMT sensitivity toward cervical SC pathologic changes in MS, thereby inspiring future work to use this technique for MR imaging monitoring and as a tool in remyelination therapeutic strategies. Meanwhile, further investigations should be directed toward confirming this higher sensitivity in different cohorts and further evaluation of the specificity of ihMT to these myelin-related pathologic changes.

\section{CONCLUSIONS}

The use of a multiparametric MR imaging protocol combined with an automatic template-based GM/WM segmentation approach in the current study outlined a higher sensitivity of the ihMT technique toward SC pathophysiologic changes in MS compared with atrophy measurements, DTI, and conventional MT, presumably 
due to its supposed higher specificity to myelin-content variations. The clinical correlations between ihMTR and functional impairment observed in patients with MS also argue for its potential clinical relevance, paving the way for future longitudinal multicentric clinical trials in MS.

\section{ACKNOWLEDGMENTS}

The authors thank V. Gimenez and L. Pini for study logistics, A. Rico for patient recruitment, and S. Gherib for brain lesion segmentation.

Disclosures: Jean Pelletier-UNRELATED: Board Membership: Biogen, Teva Pharmaceutical Industries, Novartis, Merck, MedDay Pharma, Roche; Grants/ Grants Pending: Roche, Merck; Travel/Accommodations/Meeting Expenses Unrelated to Activities Listed: Biogen, Roche, Teva Pharmaceutical Industries, Merck, MedDay. Virginie Callot—RELATED: Grant: Fondation pour l'Aide à la Recherche sur la Sclérose En Plaques, Comments: The money helped for MR imaging exploration.* *Money paid to the institution.

\section{REFERENCES}

1. Thompson AJ, Banwell BL, Barkhof F, et al. Diagnosis of multiple sclerosis: 2017 revisions of the McDonald criteria. Lancet Neurol 2018;17:162-73 CrossRef Medline

2. Gass A, Rocca MA, Agosta F, et al; MAGNIMS Study Group. MRI monitoring of pathological changes in the spinal cord in patients with multiple sclerosis. Lancet Neurol 2015;14:443-54 CrossRef Medline

3. Kearney H. Clinical monitoring of multiple sclerosis should routinely include spinal cord imaging: No. Mult Scler 2018;24:1537-39 CrossRef Medline

4. Stroman PW, Wheeler-Kingshott C, Bacon M, et al. The current state-of-the-art of spinal cord imaging: methods. Neuroimage 2014;84:1070-81 CrossRef Medline

5. Basser PJ, Mattiello J, LeBihan D. MR diffusion tensor spectroscopy and imaging. Biophys J 1994;66:259-67 CrossRef Medline

6. Wolff SD, Balaban RS. Magnetization transfer contrast (MTC) and tissue water proton relaxation in vivo. Magn Reson Med 1989;10: 135-44 CrossRef Medline

7. Zackowski KM, Smith SA, Reich DS, et al. Sensorimotor dysfunction in multiple sclerosis and column-specific magnetization transferimaging abnormalities in the spinal cord. Brain 2009;132:1200-09 CrossRef Medline

8. Oh J, Cybulsky K, Chen M, et al. Longitudinal changes in quantitative spinal cord MRI in multiple sclerosis patients: results of a 5year study (S47.001). Neurology Apr 2018, 90:(15 Supplement) S47.001. https://n.neurology.org/content/90/15_Supplement/S47.001. Accessed May 10, 2019

9. Mottershead JP, Schmierer K, Clemence M, et al. High field MRI correlates of myelin content and axonal density in multiple sclerosis. J Neurol 2003;250:1293-1301 CrossRef Medline

10. O’Muircheartaigh J, Vavasour I, Ljungberg E, et al. Quantitative neuroimaging measures of myelin in the healthy brain and in multiple sclerosis. Hum Brain Mapp 2019;40:2104-16 CrossRef Medline

11. Heath F, Hurley SA, Johansen-Berg H, et al. Advances in noninvasive myelin imaging. Dev Neurobiol 2018;78:136-51 CrossRef Medline

12. Mackay A, Whittall $\mathrm{K}$, Adler J, et al. In vivo visualization of myelin water in brain by magnetic resonance. Magn Reson Med 1994;31: 673-77 CrossRef Medline

13. Alonso-Ortiz E, Levesque IR, Pike GB. MRI-based myelin water imaging: a technical review. Magn Reson Med 2015;73:70-81 CrossRef Medline

14. Sled JG, Pike GB. Quantitative imaging of magnetization transfer exchange and relaxation properties in vivo using MRI. Magn Reson Med 2001;46:923-31 CrossRef Medline
15. Smith AK, Dortch RD, Dethrage LM, et al. Rapid, high-resolution quantitative magnetization transfer MRI of the human spinal cord. Neuroimage 2014;95:106-16 CrossRef Medline

16. Schmierer K, Tozer DJ, Scaravilli F, et al. Quantitative magnetization transfer imaging in postmortem multiple sclerosis brain. $J$ Magn Reson Imaging 2007;26:41-51 CrossRef Medline

17. Varma G, Girard OM, Prevost VH, et al. In vivo measurement of a new source of contrast, the dipolar relaxation time, $T_{1 D}$, using a modified inhomogeneous magnetization transfer (ihMT) sequence. Magn Reson Med 2017;78:1362-72 CrossRef Medline

18. Girard OM, Callot V, Prevost VH, et al. Magnetization transfer from inhomogeneously broadened lines (ihMT): improved imaging strategy for spinal cord applications. Magn Reson Med 2017;77:581-91 CrossRef Medline

19. Varma G, Duhamel G, Bazelaire C, et al. Magnetization transfer from inhomogeneously broadened lines: a potential marker for myelin. Magn Reson Med 2015;73:614-22 CrossRef Medline

20. Van Obberghen E, Mchinda S, Le Troter A, et al. Evaluation of the sensitivity of inhomogeneous magnetization transfer (ihMT) MRI for multiple sclerosis. AJNR Am J Neuroradiol 2018;39:63441 CrossRef Medline

21. Taso M, Girard OM, Duhamel G, et al. Tract-specific and agerelated variations of the spinal cord microstructure: a multi-parametric MRI study using diffusion tensor imaging (DTI) and inhomogeneous magnetization transfer (ihMT). NMR Biomed 2016;29: 817-32 CrossRef Medline

22. Rasoanandrianina H, Grapperon AM, Taso M, et al. Region-specific impairment of the cervical spinal cord (SC) in amyotrophic lateral sclerosis: a preliminary study using SC templates and quantitative MRI (diffusion tensor imaging/inhomogeneous magnetization transfer). NMR Biomed 2017;30:e3801 CrossRef Medline

23. Polman CH, Reingold SC, Banwell B, et al. Diagnostic criteria for multiple sclerosis: 2010 revisions to the McDonald criteria. Ann Neurol 2011;69:292-302 CrossRef Medline

24. Compston A. Aids to the investigation of peripheral nerve injuries: Medical Research Council-Nerve Injuries Research Committee. His Majesty's Stationery Office: 1942; pp. 48 (iii) and 74 figures and 7 diagrams; with aids to the examination of the peripheral nervous system. By Michael O'Brien for the Guarantors of Brain. Saunders Elsevier: 2010; pp. [8] 64 and 94 figures. Brain 2010; 133:2838-44 CrossRef Medline

25. Allgöwer $\mathrm{K}$, Kern $\mathrm{C}$, Hermsdörfer J. Predictive and reactive grip force responses to rapid load increases in people with multiple sclerosis. Arch Phys Med Rehabil 2017;98:525-33 CrossRef Medline

26. Cutter GR, Baier ML, Rudick RA, et al. Development of a multiple sclerosis functional composite as a clinical trial outcome measure. Brain 1999;122:871-82 CrossRef Medline

27. De Leener B, Lévy S, Dupont SM, et al. SCT: Spinal Cord Toolbox, an open-source software for processing spinal cord MRI data. NeuroImage 2017;145:24-43 CrossRef Medline

28. Fonov VS, Le Troter A, Taso M, et al. Framework for integrated MRI average of the spinal cord white and gray matter: the MNIPoly-AMU template. Neuroimage 2014;102(Pt 2):817-87 CrossRef Medline

29. Taso M, Le Troter A, Sdika M, et al. A reliable spatially normalized template of the human spinal cord: applications to automated white matter/gray matter segmentation and tensor-based morphometry (TB1000) mapping of gray matter alterations occurring with age. Neuroimage 2015;117:20-28 CrossRef Medline

30. Jenkinson M, Beckmann CF, Behrens TE, et al. FSL. Neuroimage 2012;62:782-90 CrossRef Medline

31. De Leener B, Kadoury S, Cohen-Adad J. Robust, accurate and fast automatic segmentation of the spinal cord. Neuroimage 2014;98: 528-36 CrossRef Medline

32. Lévy S, Benhamou M, Naaman C, et al. White matter atlas of the human spinal cord with estimation of partial volume effect. Neuroimage 2015;119:262-71 CrossRef Medline 
33. De Leener B, Fonov VS, Collins DL, et al. PAM50: unbiased multimodal template of the brainstem and spinal cord aligned with the ICBM152 space. Neuroimage 2018;165:170-79 CrossRef Medline

34. Kearney H, Schneider T, Yiannakas MC, et al. Spinal cord grey matter abnormalities are associated with secondary progression and physical disability in multiple sclerosis. I Neurol Neurosurg Psychiatry 2015;86:608-14 CrossRef Medline

35. Prados F, Cardoso MJ, Yiannakas MC, et al. Fully automated grey and white matter spinal cord segmentation. Sci Rep 2016;6:36151 CrossRef Medline

36. Datta E, Papinutto N, Schlaeger R, et al. Gray matter segmentation of the spinal cord with active contours in MR images. Neuroimage 2017;147:788-99 CrossRef Medline

37. Schlaeger R, Papinutto N, Panara V, et al. Spinal cord gray matter atrophy correlates with multiple sclerosis disability. Ann Neurol 2014;76:568-80 CrossRef Medline

38. Zeydan B, Gu X, Atkinson EJ, et al. Cervical spinal cord atrophy: an early marker of progressive MS onset. Neurol Neuroimmunol Neuroinflamm 2018;5:e435 CrossRef Medline

39. Valsasina P, Aboulwafa M, Preziosa P, et al. Cervical cord T1weighted hypointense lesions at MR imaging in multiple sclerosis: relationship to cord atrophy and disability. Radiology 2018;288: 234-44 CrossRef Medline

40. Oh J, Zackowski K, Chen M, et al. Multiparametric MRI correlates of sensorimotor function in the spinal cord in multiple sclerosis. Mult Scler J 2013;19:427-35 CrossRef Medline

41. Filippi M, Agosta F. Magnetization transfer MRI in multiple sclerosis. J Neuroimaging 2007;17(Suppl 1):22S-26S CrossRef Medline

42. Dvorak AV, Ljungberg E, Vavasour IM, et al. Rapid myelin water imaging for the assessment of cervical spinal cord myelin damage. Neuroimage Clin 2019;23:101896 CrossRef Medline

43. Rasoanandrianina H, Duhamel G, Massire A, et al. A new rapid and high-resolution multi-slice inhomogeneous magnetization transfer protocol to evaluate diffuse and regional cervical cord myelination at 3T. In: Proceedings of the Annual Meeting of the International Society of Magnetic Resonance in Medicine, Paris, France; June 16-21, 2018; 1855

44. Troalen T, Callot V, Varma G, et al. Cervical spine inhomogeneous magnetization transfer (ihMT) imaging using ECG-triggered 3D rapid acquisition gradient-echo (ihMT-RAGE). In: Proceedings of the Annual Meeting of the International Society for Magnetic Resonance in Medicine, Montreal, Canada; May 11-16, 2019; 0300

45. Dula AN, Pawate $\mathrm{S}$, Dortch $\mathrm{RD}$, et al. Magnetic resonance imaging of the cervical spinal cord in multiple sclerosis at 7T. Mult Scler 2016;22:320-28 CrossRef Medline

46. Massire A, Taso M, Besson P, et al. High-resolution multi-parametric quantitative magnetic resonance imaging of the human cervical spinal cord at 7T. Neuroimage 2016;143:58-69 CrossRef Medline

47. Mchinda S, Varma G, Prevost VH, et al. Whole brain inhomogeneous magnetization transfer (ihMT) imaging: sensitivity enhancement within a steady-state gradient echo sequence. Magn Reson Med 2018;79:2607-19 CrossRef Medline

48. Gros C, De Leener B, Badji A, et al. Automatic segmentation of the spinal cord and intramedullary multiple sclerosis lesions with convolutional neural networks. Neuroimage 2019;184:901-15 CrossRef Medline

49. Eden D, Gros C, Badji A, et al. Spatial distribution of multiple sclerosis lesions in the cervical spinal cord. Brain 2019;142:633-46 CrossRef Medline

50. Feiweier T, Huwer S, Kim TH, Porter DA, et al. Method and magnetic resonance system to reduce distortions in diffusion imaging. Siemens Aktiengesellschaft, assignee. United States patent 20110187367. 2011 04.08.2011. http://www.google.com/patents/US8508226. Accessed June 11, 2016

51. Jenkinson M, Bannister P, Brady M, et al. Improved optimization for the robust and accurate linear registration and motion correction of brain images. Neuroimage 2002;17:825-41 CrossRef Medline 